\title{
Skating with a Force Controlled Quadrupedal Robot
}

\section{Conference Paper}

Author(s):

Bjelonic, Marko; Bellicoso, C. Dario; Tiryaki, M. Efe; Hutter, Marco (D)

Publication date:

2018

Permanent link:

https://doi.org/10.3929/ethz-b-000278902

\section{Rights / license:}

In Copyright - Non-Commercial Use Permitted

Originally published in:

https://doi.org/10.1109/IROS.2018.8594504 


\title{
Skating with a Force Controlled Quadrupedal Robot
}

\author{
Marko Bjelonic, C. Dario Bellicoso, M. Efe Tiryaki and Marco Hutter
}

\begin{abstract}
Traditional legged robots are capable of traversing challenging terrain, but lack of energy efficiency when compared to wheeled systems operating on flat environments. The combination of both locomotion domains overcomes the trade-off between mobility and efficiency. Therefore, this paper presents a novel motion planner and controller which together enable a legged robot equipped with skates to perform skating maneuvers. These are achieved by an appropriate combination of planned reaction forces and gliding motions. Our novel motion controller formulates a Virtual Model Controller and an optimal contact force distribution which takes into account the nonholonomic constraints introduced by the skates. This approach has been tested on the torque-controllable robot ANYmal equipped with passive wheels and ice skates as end-effectors. We conducted experiments on flat and inclined terrain, whereby we show that skating motions reduces the cost of transport by up to $80 \%$ with respect to traditional walking gaits.
\end{abstract}

\section{INTRODUCTION}

Recent research in robotic locomotion has shown a great variety of locomotion strategies. A large part of this work has been focused on traditional walking maneuvers, whereby the feet are assumed to remain stationary when in contact with the environment. However, comparisons between wheeled and legged robots have shown that the former are much superior on flat terrain compared to legged robots regarding the energy efficiency [1]. The combination of wheels and legs, if properly implemented, can be exploited to take advantage of both locomotion domains.

In this paper, we describe the planning and control framework which enable a quadrupedal robot to perform skating ${ }^{1}$ motions using passive wheels and on ice as depicted in Fig.11.

\section{A. Related Work}

Combining walking and driving has been an active research topic in recent years. In particular, actuated wheels have proven to be a great extension to increase the efficiency of the locomotion while maintaining the rough terrain capabilities by lifting the robot's legs [4]-[8]. However, most of the robots using actuated wheels are not combining walking and rolling simultaneously. The legs typically act as a suspension system while driving and the wheels are

This work has been conducted as part of ANYmal Research, a community to advance legged robotics.

This work was supported in part by the Swiss National Science Foundation (SNF) through the National Centres of Competence in Research Robotics and Digital Fabrication.

All authors are with the Robotic Systems Lab, ETH Zürich, 8092 Zürich, Switzerland, email: firstname.surname@mavt.ethz.ch

${ }^{1}$ We define skating as the action or activity of gliding on ice skates or roller skates.

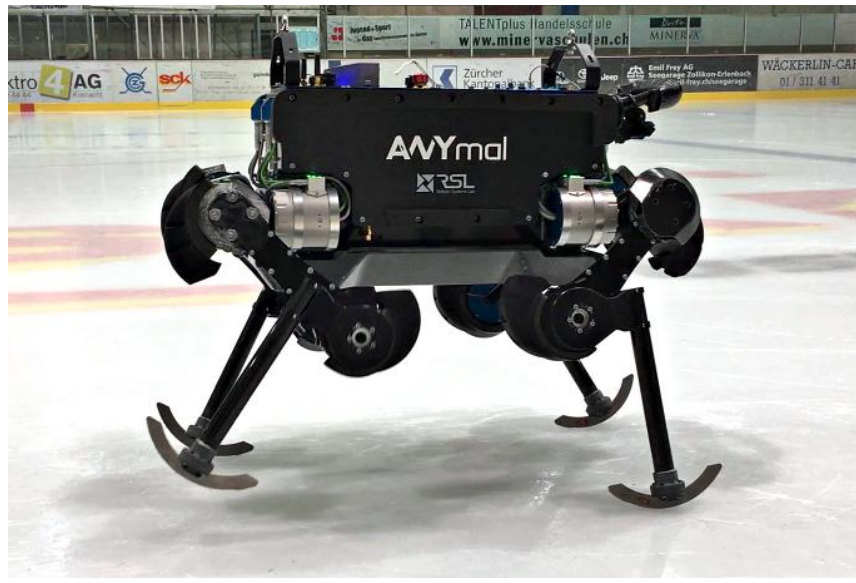

Fig. 1. The robot ANYmal [2], [3] performing skating motions on ice.

fixed during walking to overcome obstacles. One exception is Boston Dynamics' wheeled-legged robot Handle that is able to jump and drive at the same time to dynamically overcome high obstacles [9]. However, there are no published papers about Handle, and as such, there is no knowledge about Boston Dynamics' locomotion framework. In addition, only some of the works explore force control for wheeledlegged locomotion although this is a widely used approach in traditional legged locomotion [2], [10]-[12]. Most of the existing literature focuses on actuated wheels. Skating locomotion, on the other hand, poses a solution to decrease the energy consumption when overcoming long, sufficiently even distances with relatively low costs for hardware modifications.

Most of the literature regarding skating robots can be divided into three categories: design of the skating robot, motion planning, and motion control of skating motions. Related to our work there is some work that focused on skating motions for quadrupedal robots. The quadrupedal robot Robosimian of NASA's Jet Propulsion Laboratory (JPL) uses six degrees of freedom (DOF) to set the pose of each skate [13]. There is no force feedback available during skating and, as such, the robot executes open-loop joint position trajectories that are tracked by a PID controller without taking into account disturbances and drifting states. By this means, the motion plan given by the joint trajectories is not updated during locomotion. In addition, the motion plan is designed in such a way that the legs never lift off, i.e., the skates always stay in contact with the ground. In contrast to our work, Bellegarda et al. [13] only presented simulation results. Similarly to Robosimian, the roller-walker by Hirose and his research group [14], [15] performs skating motions without lifting its legs, and the motion is executed without 
controlling the ground reaction force. Interaction with the environment is achieved through the contact points which impose changing contact constraints and interaction forces. Without force control, the contact constraints are prone to be violated, and the locomotion is not robust against unpredicted terrain irregularities.

Humanoids on passive wheels have been explored by some research labs [16]-[18]. Most of the literature showed static stability where both skates are always in contact, and the control approach is based on position controlled joints. The authors of [19] have shown similar results for a small sized humanoid robot that was performing skating motions on ice. The work in [20] showed skating motions with the humansized humanoid robot HRP-2 balancing on a skateboard. In this work, the robot uses a zero moment point (ZMP) approach for motion planning, and the robot controls the contact force on the ground to push with one leg and to maintain balance.

\section{B. Contribution}

This paper presents skating motions with a force controlled quadrupedal robot which combines the advantages wheeled and legged locomotion. We introduce a motion planner together with a motion controller that is novel in such a way that the supporting legs are fully torque controlled during the skating motion. Our main contribution is a novel virtual model controller (VMC) with an optimal contact force distribution that takes into account the nonholonomic contact constraints of the skates by introducing a friction triangle. Finally, to verify our skating motions, we show experimental results on the robot ANYmal [2]. To the best of our knowledge, this is the first time skating motions are shown on a fully torque-controllable quadrupedal robot.

\section{MECHANICAL DESIGN}

The robot ANYmal is constructed from a single torso, i.e., free-floating base, and twelve identical hinged joints actuated by torque controlled series-elastic actuators [3]. Each leg is composed of three consecutive joints arranged as hip adduction/abduction (HAA), hip flexion/extension (HFE) and knee flexion/extension (KFE). This kinematic topology allows controlling three DOF, i.e., if the end-effector position is fixed, the orientation can not be changed.

Motion studies analyzed the gliding and pushing motion of male and female Olympic speed skaters [21]. The study revealed that the forward motion of human ice skaters is generated by placing the skates laterally with respect to (w.r.t.) the forward direction and by generating a push-off force in the lateral direction w.r.t. the gliding direction of the skate. Thanks to the kinematic redundancy of human legs, both the position and the orientation of the skates can be independently controlled. It is not possible to achieve such kind of skating motions for our quadrupedal robot ANYmal without introducing additional actuated joints. Therefore, we decided to imitate a less conventional approach for skating, which is performed by figure skaters using claws attached in the front of the skates. The claw is used to exert a push

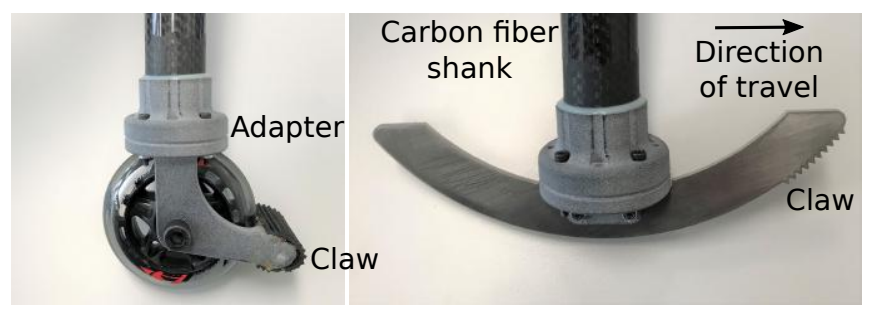

Fig. 2. The design of the rollerblades for one leg (left) consists of one wheel with a radius of $4 \mathrm{~cm}$ and the contact surface of the claw consists of a rubber profile. The design of the ice skates (right) consists of a single blade with a radius of $12 \mathrm{~cm}$ and the claw is inspired by the ice skates of figure skaters.

off force on the ground with one skate while gliding on the others.

To avoid any additional mechanical complexity, a skate design with only one passive wheel or one single blade per leg is used (see Fig. 2). The claws are equipped on the skates in such a way that they do not touch the ground during the gliding motion. While performing a pushing motion, the claws make contact with the ground, and the robot is able to exert ground reaction forces in all directions. The estimated location of the claw, when in contact, is obtained from forward kinematics. The gliding direction of the skates is pointing towards the forward direction of the torso. Moreover, the yaw angle of the gliding direction equals the yaw angle of the torso due to the HAA-HFE-KFE design of the legs. With this leg design, the robot is able to perform small curvatures by rotating the torso around the yaw axis, and for large curvatures, the robot repositions its legs.

\section{MOTION PLANNING}

As highlighted in Section II] the robot performs pushing motions by moving its legs such that the claws establish contact with the ground. Fig. 3 gives an overview of the pushing motion of the robot ANYmal on rollerblades. The following section elaborates how these motions are generated.

\section{A. Terrain Estimation and Adaptation}

As done in [12], the terrain on which the robot is blindly locomoting is approximated by a free rotating plane fitted through the most recent contact locations using a leastsquares method. The contact state of each leg, i.e., whether the end-effector is in contact or not with the terrain, is determined by thresholding an estimation of the force acting on it [22]. In contrast to point contacts, the estimation of the contact location is non-trivial due to the shape of the skates and the changing contact location during locomotion. For this reason, we assume locomotion on flat terrain. This is a valid assumption on smooth skating fields as well as on artificial ice rinks. The contact point is calculated by taking into account the shape of the skate and the estimated terrain plane. The position vector from the torso to the contact point is defined by ${ }_{B} \mathbf{r}_{B W_{i}} \in \mathbb{R}^{3}$, where the term denotes the coordinates of the vector $\mathbf{r}_{B W_{i}}$ in the Euclidean space $\mathbb{E}^{3}$ from base frame $B$ to contact frame $W_{i}$ of leg $i$, represented in the coordinate system of the base frame $B$ [23]. 


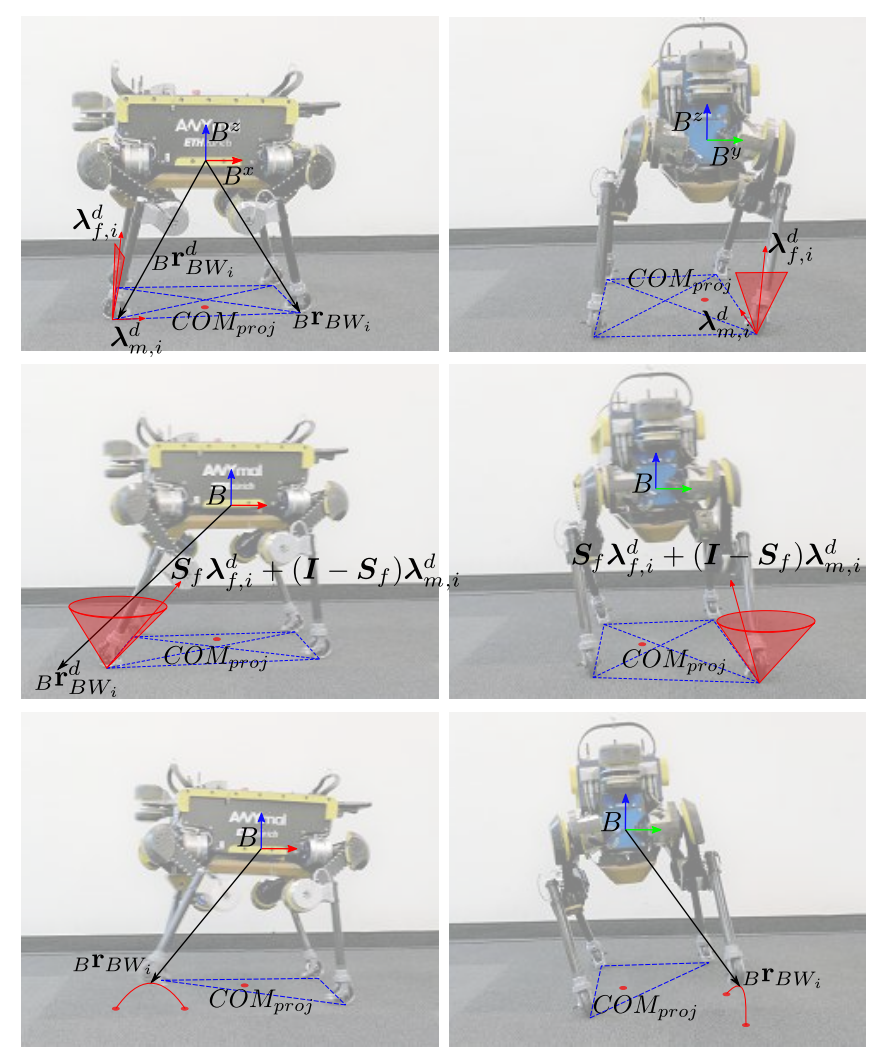

Fig. 3. The robot ANYmal [3] on rollerblades performs a pushing motion with the right hind leg, where the front, hind, right and left legs are defined based on the robot's base frame $B$. The figure shows a time sequence (up to down) of the robot's pushing motion from two different viewpoints, i.e., side view (left column) and back view (right column). First row: The robot's COM is moved towards the pushing leg. By this means, the pushing leg is able to create a higher normal force due to the weight of the torso, and this helps to stay inside the frictions constraint during the pushing motion. The contact constraint in this phase is defined by a friction triangle (red triangle) due to the nonholonomic constraints of the wheels. Moreover, the motion is controlled through the two constraint forces $\boldsymbol{\lambda}_{f}$ and $\boldsymbol{\lambda}_{m}$ as explained in Section IV Middle row: During the pushing motion the contact constraint switches to a friction cone due to the claws at the skate (See Section III). The pushing motion is achieved by defining the next desired foothold ${ }_{B} \mathbf{r}_{B W_{i}}^{d}$ to the back. In addition, the motion planner commands the COM to move to the next support polygon. Last row: After executing the pushing motion the robot swings back to its starting position given by ${ }_{B} \mathbf{r}_{B W_{i}}^{d}$ of the first row. This motion is afterward repeated for the left hind leg.

We define a so-called Control frame $C$ [24] whose z-axis is aligned with the estimated terrain normal and whose $\mathrm{x}$-axis is perpendicular to the estimated terrain normal and aligned with the heading direction of the robot. The heading direction is oriented according to the average positions of the front and hind skate positions. Moreover, the origin of $C$ is located at the inertial frame $I$. The definition of such a frame allows to automatically align the motion of the robot to tilted terrain by setting an identity orientation reference w.r.t. it.

\section{B. Gait Pattern}

A gait pattern defines the contact schedule by specifying lift-off and touch-down events for all legs. For each of the latter, we split the planned motion into a stance and swing phase, which describes the role of the leg between different contact events. Additionally, the contact schedule splits up the stance phase into a glide and push phase.
The latter schedules the leg to execute a force task, which keeps contact with the ground while pushing the robot in the desired gliding direction ${ }^{2}$ We employ symmetrical gaits for the skating motions with predefined lift-off, touch-down and pushing time durations. The quadrupedal robot with its mechanical design described in Section $\Pi$ is able to skate in the forward and backward direction thanks to its symmetrical design. During the forward skating motion the two hind legs are switching between stance, push and swing phase, while the two front legs are scheduled to remain in stance configuration (see Fig. 3).

\section{Motion Generation of the Torso}

To ensure balance at all times, the reference torso motion is planned such that the COM of the robot projected onto the ground lies inside the convex hull of the contact points, or support polygon. To apply a pushing force, which accelerates the robot, the normal force of the pushing leg needs to be high enough such that the resultant ground reaction force stays inside the friction cone. The normal force is increased by moving the COM towards the leg that is supposed to start the pushing motion (see Fig. 3). Due to the weight of the torso, the normal force increases at the pushing leg. To keep balance after the pushing motion, the COM needs to move towards the next support polygon before the pushing leg lifts off. We use quintic polynomial splines to define the motion of the torso [12]. The height of the robot is kept constant during the motion.

Since the legs in contact are supporting the motion of the torso, their desired motion is defined by the desired base motion. However, this is only valid for the directions in which the skate is able to generate ground reaction forces. In the gliding direction, the skate is not able to generate any ground reaction force which could move the torso in a certain direction. In our case, the robot can only generate forces in the $y$ and $z$ direction of the torso frame $B$ (see Fig. 3).

\section{Motion Generation of Stance and Swing Legs}

During the gliding phase, each leg $i$ stays at a desired position ${ }_{B} \mathbf{r}_{B W_{i}}^{d} \in \mathbb{R}^{3}$, where the superscript $d$ indicates a desired value. The primary task during the pushing phase is to establish contact with the claw such that the leg is able to create ground reaction forces in all directions. Due to the kinematics of the leg and the claw position, this is achieved by moving back the leg along the gliding direction. The applied ground reaction forces of the pushing leg are controlled through a spring-damper system as explained in Section IV The start and end position of the leg is interpolated using a predefined pushing time to generate a reference trajectory for the spring-damper system. This enables the robot to create a pushing force that moves the torso along the gliding direction. After executing the pushing motion the leg swings back to its nominal contact position.

\footnotetext{
${ }^{2}$ The gliding direction is defined to coincide with the rolling/sliding direction of the skates, i.e., the gliding direction of ANYmal coincides with the projected forward direction of the torso onto the estimated terrain plane in Section III-A
} 
A sequence of quintic splines represents the motion plan for the swing trajectory.

\section{MOTION CONTROLLER}

Given the desired torso and skate trajectories, the motion controller tracks them by computing torque, angular position and velocity references for each of the joints. We use a force control approach for the legs in contact and position control for the legs during the swing motion.

\section{A. Motion Execution of the Torso and Stance Legs}

Our main contribution is the following VMC [11] and an optimal contact force distribution [12] that takes into account the nonholonomic constraints of the skates. The VMC outputs a reference force ${ }_{C} \mathbf{f}^{d} \in \mathbb{R}^{3}$ and torque ${ }_{C} \mathbf{t}^{d} \in \mathbb{R}^{3}$ which are designed as a virtual spring-damper acting at the torso and are computed as a function of the desired motion described in Section III. The controller is defined by

$$
\begin{aligned}
{ }_{C} \mathbf{f}^{d}= & \mathbf{K}_{p}^{f}\left({ }_{C} \mathbf{r}_{I B}^{d}-{ }_{C} \mathbf{r}_{I B}\right)+\mathbf{K}_{i}^{f} \int\left({ }_{C} \mathbf{r}_{I B}^{d}-{ }_{C} \mathbf{r}_{I B}\right) \\
& +\mathbf{K}_{d}^{f}\left({ }_{C} \mathbf{v}_{B}^{d}-{ }_{C} \mathbf{v}_{B}\right)+m_{C} \mathbf{a}_{B}-\sum_{k \in \mathcal{B}}{ }_{C} \mathbf{f}_{k}^{g} \\
{ }_{C} \mathbf{t}^{d}= & \mathbf{K}_{p}^{t}\left(\mathbf{q}_{I B}^{d} \boxminus \mathbf{q}_{I B}\right)+\mathbf{K}_{i}^{t} \int\left(\mathbf{q}_{I B}^{d} \boxminus \mathbf{q}_{I B}\right) \\
& +\mathbf{K}_{d}^{t}\left({ }_{C} \boldsymbol{\omega}_{I B}^{d}-{ }_{C} \boldsymbol{\omega}_{I B}\right)-\sum_{k \in \mathcal{B}}{ }_{C} \mathbf{r}_{I S_{k}} \times{ }_{C} \mathbf{f}_{k}^{g},
\end{aligned}
$$

where ${ }_{C} \mathbf{r}_{I B} \in \mathbb{R}^{3},{ }_{C} \mathbf{v}_{B} \in \mathbb{R}^{3},{ }_{C} \mathbf{a}_{B} \in \mathbb{R}^{3}, \mathbf{q}_{I B} \in S O(3)$, ${ }_{C} \boldsymbol{\omega}_{I B} \in \mathbb{R}^{3}$ are position, velocity, acceleration, rotation quaternion and angular velocity of the torso, respectively. $\mathcal{B}$ is the set of bodies of each link, ${ }_{C} \mathbf{r}_{I S_{k}}$ is the position of the COM of each body $k,{ }_{C} \mathbf{f}_{k}^{g} \in \mathbb{R}^{3}$ is the gravitational force acting on body $k, \boxminus$ is the box-minus operator [23]. Moreover, the motion is described w.r.t. the Control frame $C$. The tracking of the base motion is achieved by integrating feed-forward terms such as gravity compensation and reference acceleration, as well as feed-back terms which encode deviation of the torso's estimated pose and twist from the reference motion through diagonal gain matrices $\mathbf{K}_{p}^{f}, \mathbf{K}_{d}^{f}$, $\mathbf{K}_{i}^{f}, \mathbf{K}_{p}^{t}, \mathbf{K}_{d}^{t}$ and $\mathbf{K}_{i}^{t}$.

The total net force and torque of the VMC are mapped by an optimal contact force distribution algorithm into the vector of constraint forces ${ }_{C} \boldsymbol{\lambda}_{f}^{d} \in \mathbb{R}^{3 n_{c}}$ at the estimated contact point of each support leg, with $n_{c}$ the number of legs in contact. To this end, we need to take into account the nonholonomic constraints. As opposed to the case of point feet, the friction constraints change for skates along the gliding direction. Instead of approximating the friction cones with pyramids [12], the contact constraints of each leg $i$ need to be defined by a friction triangle $\left(\mu_{l a t}, \lambda_{N, \min }\right.$, $\left.\lambda_{N, \max }\right)$. By this means, only contact forces in the norma 3

\footnotetext{
${ }^{3}$ The normal direction is aligned with the terrain normal estimated in Section III-A
}

and latera $I^{4}$ direction are generated. This formulation results in a quadratic program given by

$$
\begin{array}{ll}
\underset{C \boldsymbol{\lambda}_{f}^{d}}{\operatorname{minimize}} & \left(\boldsymbol{A}_{C} \boldsymbol{\lambda}_{f}-\boldsymbol{b}\right)^{T} \boldsymbol{S}\left(\boldsymbol{A}_{C} \boldsymbol{\lambda}_{f}-\boldsymbol{b}\right)+{ }_{C} \boldsymbol{\lambda}_{f}^{T} \boldsymbol{W}_{C} \boldsymbol{\lambda}_{f} \\
& \underbrace{\left[\begin{array}{ccc}
\cdots & \boldsymbol{I} & \cdots \\
\cdots & { }_{C} \mathbf{r}_{B W_{i}} \times & \cdots
\end{array}\right]}_{\boldsymbol{A}} \underbrace{\left[\begin{array}{c}
\vdots \\
\boldsymbol{\lambda}_{f, i} \\
\vdots
\end{array}\right]}_{C \boldsymbol{\lambda}_{f}}=\underbrace{\left[\begin{array}{c}
C^{\mathbf{f}^{d}} \\
\mathbf{t}^{d}
\end{array}\right]}_{\boldsymbol{b}} \\
\text { subject to } & \left\|{ }_{C} \boldsymbol{\lambda}_{f, l a t, j}\right\| \leq \mu_{l a t} \lambda_{N, j} \\
& \left\|\boldsymbol{\lambda}_{f, g l i d e, j}\right\|=0 \\
& \left.\left\|{ }_{C} \boldsymbol{\lambda}_{f, T, l}\right\| \leq \mu_{T} \lambda_{N, l}\right\} \forall \text { gliding legs } j \\
& \lambda_{N, i} \geq \lambda_{N, \text { min }} \\
& \lambda_{N, i} \leq \lambda_{N, \text { max }},
\end{array}
$$

where $S$ is a diagonal weighting matrix used to penalize different components of the cost function, $\boldsymbol{W}$ is a diagonal matrix used as a regularizer to minimize the contact forces, $\mu_{\text {lat }}$ and $\mu_{T}$ are estimated friction coefficients, and $\lambda_{N, i} \in$ $\mathbb{R}$ is the normal component of ${ }_{C} \boldsymbol{\lambda}_{f, i}$. Here, ${ }_{C} \boldsymbol{\lambda}_{f, \text { glide, } j} \in$ $\mathbb{R}^{3}$ and ${ }_{C} \boldsymbol{\lambda}_{f, l a t, j} \in \mathbb{R}^{3}$ are the constraint forces for each gliding leg $j$ projected onto the gliding and lateral direction, respectively. In contrast, ${ }_{C} \boldsymbol{\lambda}_{f, T, l} \in \mathbb{R}^{3}$ ist the constraint force for each pushing leg $l$ projected onto the estimated terrain.

Due to the gliding constraint, the contact force distribution in (2) results in an uncontrolled motion of the contact point along the gliding direction. In order to control the motion of the contact point along the gliding direction, we add a generalized spring-damper system to the contact position by defining a vector of constraint forces for the gliding motion ${ }_{C} \boldsymbol{\lambda}_{m}^{d} \in \mathbb{R}^{3 n_{c}}$ of each leg $i$. This motion tracking task through constraint forces is defined by

$$
\begin{aligned}
{ }_{C} \boldsymbol{\lambda}_{m, \text { glide }, i}^{d}= & -k_{p}\left(\operatorname{proj}_{C} \mathbf{s}\left({ }_{C} \mathbf{r}_{B W_{i}}^{d}-{ }_{C} \mathbf{r}_{B W_{i}}\right)\right) \\
& -k_{d}\left(\operatorname{proj}_{C} \mathbf{s}\left({ }_{C} \mathbf{v}_{W_{i}}^{d}-{ }_{C} \mathbf{v}_{W_{i}}\right)\right),
\end{aligned}
$$

where ${ }_{C} \boldsymbol{\lambda}_{m, \text { glide }, i}^{d} \in \mathbb{R}^{3},{ }_{C} \mathbf{v}_{W_{i}} \in \mathbb{R}^{3}, k_{p}, k_{d}$ and $\operatorname{proj}_{C} \mathbf{s}(\mathbf{x}) \in \mathbb{R}^{3}$ are the constraint forces for each leg $i$ along the gliding direction, the velocity of the contact point, the proportional gain, the derivative gain and the projection of a vector $\mathbf{x}$ onto the gliding vector, respectively. Finally, the motion tracking of the torso and the gliding legs in ground contact is achieved through ${ }_{C} \boldsymbol{\lambda}_{f}^{d}$ and ${ }_{C} \boldsymbol{\lambda}_{m}^{d}$. The desired joint torques $\tau^{d} \in \mathbb{R}^{n_{j}}$, with $n_{j}$ the number of joints, are generated by Jacobian-transposed mapping

$$
\begin{aligned}
\boldsymbol{\tau}^{d}= & -\sum_{i \in \mathcal{I}(\boldsymbol{q})} \boldsymbol{J}_{W_{i}}^{T}\left(\boldsymbol{S}_{f C} \boldsymbol{\lambda}_{f, i}^{d}+\left(\boldsymbol{I}-\boldsymbol{S}_{f}\right)_{C} \boldsymbol{\lambda}_{m, i}^{d}\right) \\
& -\sum_{k \in \mathcal{B}} \boldsymbol{J}_{k}^{T} \mathbf{f}_{k}^{g}
\end{aligned}
$$

where $\mathcal{I}(\boldsymbol{q}), \boldsymbol{J}_{W_{i}}=\partial_{C} \boldsymbol{r}_{B W_{i}} / \partial \boldsymbol{q} \in \mathbb{R}^{3 \times n_{q}}$ (with $n_{q}$ the number of generalized positions) and $\boldsymbol{J}_{k}=\partial_{C} \boldsymbol{r}_{B B_{k}} / \partial \boldsymbol{q} \in$ $\mathbb{R}^{3 \times n_{q}}$ are the set of contacts, the constraint Jacobian of

\footnotetext{
${ }^{4}$ The lateral direction is defined to be orthogonal w.r.t. the gliding direction and the terrain normal estimated in Section III-A
} 
each contact point $W_{i}$ and the Jacobian of each body $k$, respectively. $\boldsymbol{S}_{f} \in \mathbb{R}^{3 \times 3}$ is a selection matrix that maps ${ }_{C} \boldsymbol{\lambda}_{f, i}^{d}$ onto a vector such that the entry in direction of the gliding direction is zero. This implies that $\left(\boldsymbol{I}-\boldsymbol{S}_{f}\right)_{C} \boldsymbol{\lambda}_{m, i}^{d}$ is only acting along the gliding direction (see Fig. 3). As stated in Section II the gliding direction in our case is always parallel to the projected forward direction of the torso onto the estimated terrain plane in Section III-A and since (4) is defined w.r.t. the control frame $C, \boldsymbol{S}_{f}=\operatorname{diag}(0,1,1)$.

\section{B. Motion Execution of Swing Legs}

To track the motion of the legs that are swinging towards a reference contact position, a joint-space motion controller is used which tracks joint positions and velocities obtained through inverse kinematics of Cartesian space reference states. We use an iterative inverse kinematics approach [25] to obtain the joint positions.

\section{EXPERIMENTAL RESULTS}

In this section, we demonstrate the validity of our approach through experiments on the real system. During these tests, the robot was driven by means of external velocity inputs coming from an operator joystick. All computation was carried on by the PCs integrated into the robot. A vided 5 showing the results accompanies this paper.

The following experiments rely on an accurate and robust estimation of the robot's position, velocity, and orientation w.r.t. an inertial coordinate frame. As done in [26], we fuse data from an inertial measurement unit (IMU) at the torso as well as the kinematic measurements from each actuator to acquire a fast and precise state estimation of the robot. In our extended Kalman filter, we assume that the contact point of each leg is stationary w.r.t. an inertial coordinate frame. Since

${ }^{5}$ Available at https://youtu.be/fJfAWiylpxw and https://www.bbc.com/news/av/technology-43335228/rollerskating-robot-tothe-rescue the motion along the gliding direction is unobservable, we only rely on the motions that are observable, i.e., the plane perpendicular to the gliding direction.

\section{A. Motion Tracking Performance}

The time sequence in Fig. 4 shows the performance of the skating motion on a straight path and Fig. 5 evaluates the tracking performance for two gait cycles of the motion controller in Section IV given the motion plan in Section III. First, the upper plot in Fig. 5 depicts how the desired motion plan commands the torso to lean into the next pushing leg by moving the COM sideways along the $y$-direction of the torso (A). As can be seen in the two lower plots of Fig. 5, during the pushing motion the hind legs are moving backward consecutively ( $\mathrm{B}$ and $\mathrm{D}$ ) to accelerate the robot along the forward direction of the torso. Simultaneously, the torso is moving towards the next support polygon. Due to the switch

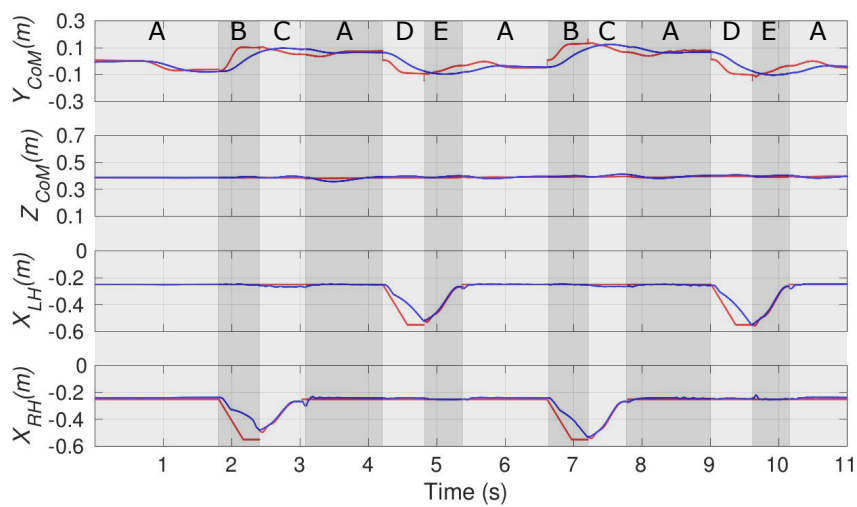

Fig. 5. The results of the skating motion depicted in Fig 4 are shown The two upper plots present the results of the motion tracking task of the COM, and the two lower plots represent the motion tracking of the legs. Moreover, the red lines are the desired motion from Section III and the blue lines represent the measured state of the robot. Two gait cycles are presented, i.e., full stance phase (A), right hind leg pushing (B), right hind leg swinging (C), left hind leg pushing (D) and left hind leg swinging (E).

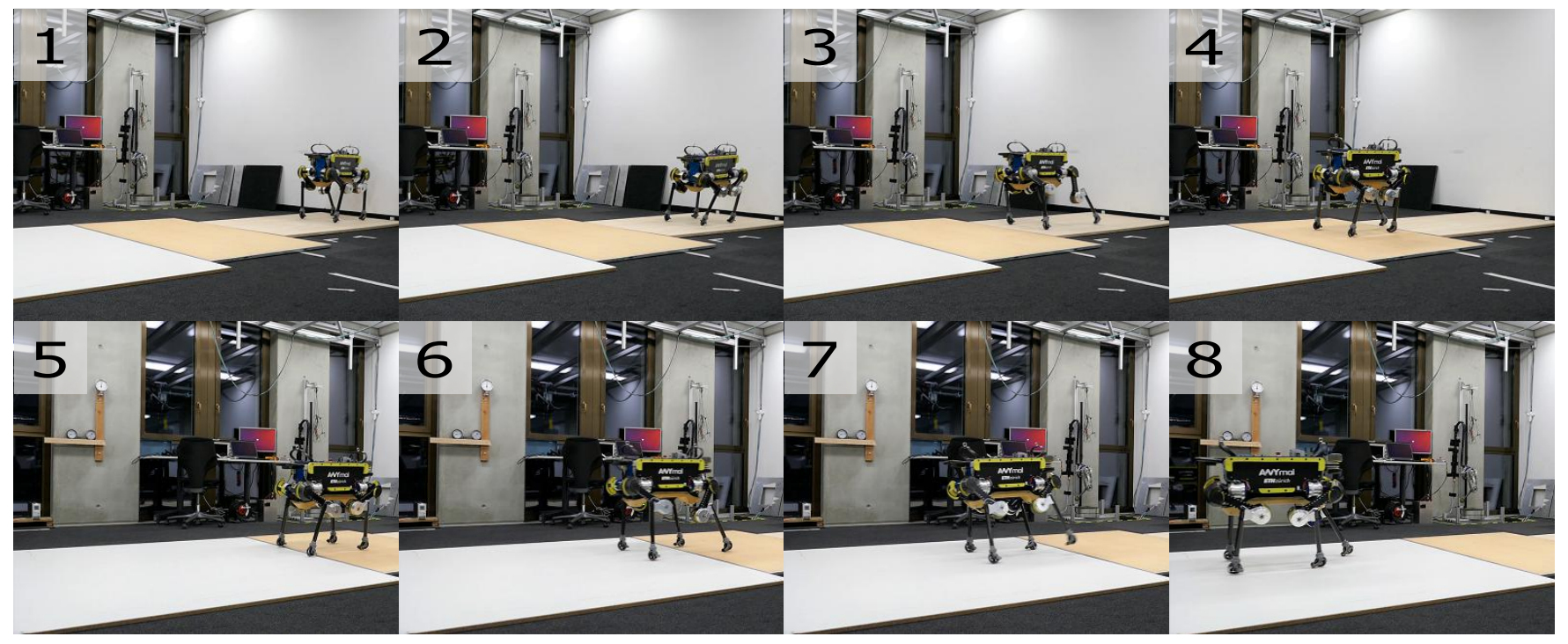

Fig. 4. The robot ANYmal performs skating motions over a distance of approximately five meters. 
TABLE I

COMPARISON OF SKATING AND WALKING LOCOMOTION OF FIVE TRIALS EACH. THE WALKING RESULTS ARE PERFORMED WITHOUT WHEELS AND WITH THE CONTROL FRAMEWORK DESCRIBED IN [22].

\begin{tabular}{cccc}
\hline Gait & Power $[\mathrm{W}]$ & Velocity $\left[\mathrm{ms}^{-1}\right]$ & COT $[-]$ \\
\hline Skating & $13.68 \pm 4.19$ & $0.342 \pm 0.027$ & $0.121 \pm 0.031$ \\
Static & $18.35 \pm 0.19$ & $0.057 \pm 0.001$ & $0.987 \pm 0.005$ \\
Trot & $50.68 \pm 4.20$ & $0.291 \pm 0.017$ & $0.598 \pm 0.001$ \\
\hline
\end{tabular}

in the contact state (free gliding to full contact at the claws) and the spring-damper system, the measured position of the legs deviates from the desired leg position slightly. Finally, after the pushing motion (B and D) the two hind legs swing back consecutively to its nominal position (C and E). The height of the torso is kept constant during the whole motion (second plot in Fig. 5.

\section{B. Cost of Transport Comparison}

To compare the performance of different locomotion approaches, the dimensionless energetic cost of transport (COT) is taken into account as a performance indicator. We use the mechanical COT to compare the performance of the skating motion with two traditional walking gaits for quadrupedal robots, i.e., static and trotting gait. The mechanical COT is defined by

$$
C O T_{\text {mech }}=\sum_{j=1}^{n} \sum_{i=1}^{4} \max \left(\tau_{j i} v_{j i}, 0\right) /\left(n m g v_{\text {avg }}\right) \text {, }
$$

where $\tau_{j i}, v_{j i}, n, m, g$ and $v_{a v g}$ are the joint torque of each sample $j$ and leg $i$, the joint velocity, the number of samples, the total mass of the robot, the gravitational acceleration and the average absolute velocity of the torso, respectively.

Table I summarizes the results from five runs with each locomotion method. The static gait lifts one leg at a time, while the trotting gait moves two legs at a time. We performed five runs with each locomotion method on the same quadrupedal robot ANYmal. The terrain consisted of flat terrain over approximately five meters (see Fig. 47). As can be seen in Table I the skating motion decreases the COT by approximately $80 \%$ w.r.t. the trotting gait and by approximately $88 \%$ w.r.t. to the static gait.

\section{Turning Motions}

In order to show that ANYmal with its three DOF per leg is able to perform turning motions, we performed skating motions while rotating the torso along the yaw direction. Due to the HAA-HFE-KFE design of each leg, the gliding direction coincides with the projected forward direction of the torso onto the terrain. Therefore, the robot is able to perform small curvatures by simply yawing the base without lifting any leg. For larger curvatures the robot needs to replace its skates. In addition, the robot is able to turn in place and move sideways by taking advantage of its legged locomotion capabilities. Fig. 6 shows the performance of the motion tracker during turning motions by comparing the

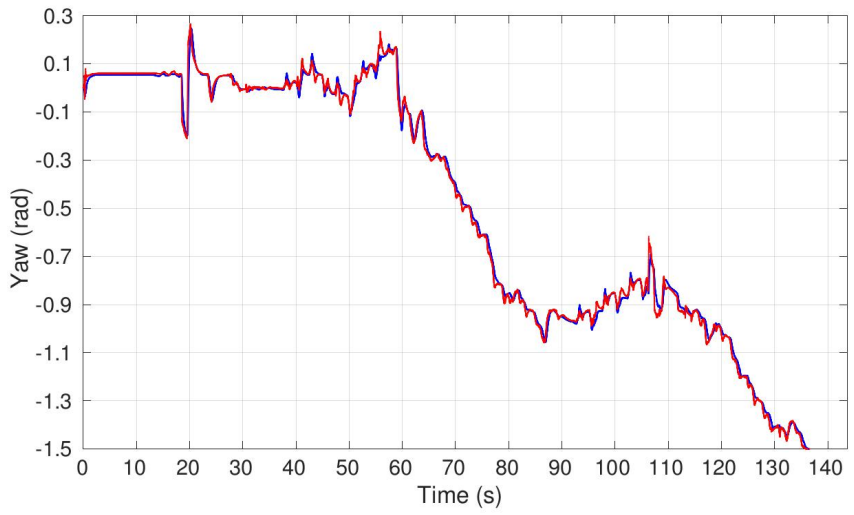

Fig. 6. The robot ANYmal changes its heading direction by rotating the torso along the yaw direction and performing stepping motions to reposition the wheels w.r.t. the torso. The results show how the motion controller tracks the desired motion plan of the yaw angle. Moreover, the red and blue line represents the desired and measured yaw angle w.r.t. the inertial frame.

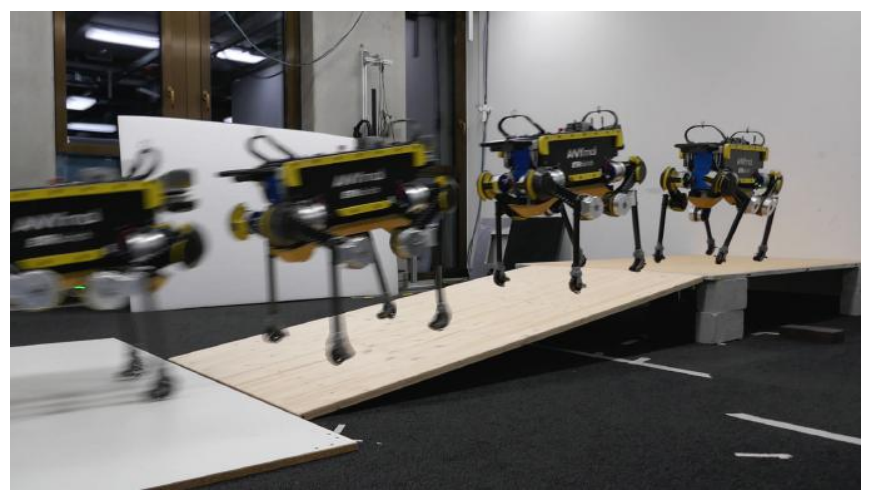

Fig. 7. The robot ANYmal performs skating motions and skates down tilted terrain with an inclination of approximately $10^{\circ}$.

measured (blue line) given by the state estimator and desired yaw angle (red line) given by the operator w.r.t. the inertial frame.

\section{Skating Motions over Inclines}

To show how the base motion adapts to tilted terrain, we performed skating motion over a $10^{\circ}$ inclination. As shown in Fig. 7. the definition of a control frame $C$ as explained in Section [III-A allows to automatically align the motion of the robot to the tilted terrain by setting an identity orientation reference of the torso w.r.t. $C$ and by using $C$ as a target frame for all desired motions, e.g., skate trajectories.

\section{DISCUSSION}

The results successfully demonstrate that the quadrupedal robot ANYmal is able to perform skating/gliding motions on flat and inclined terrain. In addition, the robot is able to turn by simply rotating the torso during gliding, by stepping or by applying a hybrid motion of both locomotion domains. The comparison of the COT between the skating motion and traditional walking gaits reveals that the skating motions significantly increase the energetic efficiency of the locomotion. The verification of this system in rough terrain is missing in this work. However, by adding a reconfigurable end-effector, which can be used for traditional walking and skating, the 
rough terrain capability of this system is straightforward to show. Previous work [22] showed that ANYmal is able to overcome challenging terrain during real-world applications by taking advantage of its legs.

\section{CONCLUSIONS}

The emphasis of this paper is on motion planning and control of skating locomotion for quadrupedal robots. The skating motion is achieved by an appropriate combination of planned reaction forces and gliding motions. Moreover, the motion plan of the torso is optimized in such a way that the normal force increases during the pushing motion, and as such, the robot is able to apply ground reaction forces which lie within the friction triangle. The plan is updated continuously at every control step, and no open-loop joint position trajectories are executed. Our novel motion controller is a VMC with an optimal contact force distribution that takes into account the nonholonomic constraint by solving a quadratic program. In contrast to most of the other work in this field, the motion controller is based on a force control approach rather than applying simple position control. The advantages of force control are the incorporation of contact constraints and the robustness against unpredicted terrain irregularities. Finally, the results on the real robot show that the skating motion reduces the COT by more than $80 \%$ w.r.t. traditional walking gaits.

One of the limiting factors in the current setup is the state estimator of the torso that is based on the assumption that the contact point of each leg is not moving during ground contact. We aim to address this by integrating visual odometry into the sensor fusion in future work. In addition, based on our first results on ice (see Fig. 1) we will further investigate the performance of the robot ANYmal performing skating motions on ice. We suggest future applications by adding a reconfigurable end-effector similar to JPLs Robosimian [13] and the roller-walker [14], [15], which can be used as either a foot or skate. By this means, the robot traverses energy efficient on flat terrain using its skates, and the robot switches to a walking configuration in rough terrain.

\section{ACKNOWLEDGMENT}

The authors would like to thank Maria Trodella, Bruno Kuster and the whole team from the artificial ice rink in Küsnacht for their support during the project.

\section{REFERENCES}

[1] M. Hutter, C. Gehring, M. A. Höpflinger, M. Blösch, and R. Siegwart, "Toward combining speed, efficiency, versatility, and robustness in an autonomous quadruped," IEEE Transactions on Robotics, 2014.

[2] M. Hutter, C. Gehring, D. Jud, A. Lauber, C. D. Bellicoso, V. Tsounis, J. Hwangbo, K. Bodie, P. Fankhauser, M. Bloesch, et al., "ANYmal - a highly mobile and dynamic quadrupedal robot," in IEEE/RSJ International Conference on Intelligent Robots and Systems (IROS), 2016.

[3] M. Hutter, C. Gehring, A. Lauber, F. Gunther, C. Bellicoso, V. Tsounis, P. Fankhauser, R. Diethelm, S. Bachmann, M. Bloesch, et al., "ANYmal - toward legged robots for harsh environments," Advanced Robotics, 2017.

[4] W. Reid, F. J. Pérez-Grau, A. H. Göktoğan, and S. Sukkarieh, "Actively articulated suspension for a wheel-on-leg rover operating on a martian analog surface," in IEEE International Conference on Robotics and Automation (ICRA), 2016.
[5] P. R. Giordano, M. Fuchs, A. Albu-Schaffer, and G. Hirzinger, "On the kinematic modeling and control of a mobile platform equipped with steering wheels and movable legs," in IEEE International Conference on Robotics and Automation, 2009.

[6] T. Klamt and S. Behnke, "Anytime hybrid driving-stepping locomotion planning," in Int. Conference on Intelligent Robots and Systems (IROS), 2017.

[7] F. Cordes, C. Oekermann, A. Babu, D. Kuehn, T. Stark, F. Kirchner, and D. R. I. C. Bremen, "An active suspension system for a planetary rover," in Proceedings of the International Symposium on Artificial Intelligence, Robotics and Automation in Space, 2014.

[8] J. Lim, I. Lee, I. Shim, H. Jung, H. M. Joe, H. Bae, O. Sim, J. Oh, T. Jung, S. Shin, et al., "Robot system of DRC-HUBO+ and control strategy of team kaist in darpa robotics challenge finals," Journal of Field Robotics, 2017.

[9] Boston Dynamics. Introducing handle. Youtube. [Online]. Available: https://www.youtube.com/watch?v=-7xvqQeoA8c

[10] C. D. Bellicoso, F. Jenelten, C. Gehring, and M. Hutter, "Dynamic locomotion through online nonlinear motion optimization for quadrupedal robots," IEEE Robotics and Automation Letters, 2018.

[11] J. Pratt, P. Dilworth, and G. Pratt, "Virtual model control of a bipedal walking robot," in IEEE International Conference on Robotics and Automation (ICRA), 1997.

[12] C. Gehring, S. Coros, M. Hutler, C. D. Bellicoso, H. Heijnen, R. Diethelm, M. Bloesch, P. Fankhauser, J. Hwangbo, M. Hoepflinger, et al., "Practice makes perfect: An optimization-based approach to controlling agile motions for a quadruped robot," IEEE Robotics \& Automation Magazine, 2016.

[13] G. Bellegarda, K. van Teeffelen, and K. Byl, "Design and evaluation of skating motions for a dexterous quadruped," in IEEE International Conference on Robotics and Automation, 2018.

[14] G. Endo and S. Hirose, "Study on roller-walker-adaptation of characteristics of the propulsion by a leg trajectory," in IEEE/RSJ International Conference on Intelligent Robots and Systems (IROS), 2008.

[15] _ "Study on roller-walker-improvement of locomotive efficiency of quadruped robots by passive wheels," Advanced Robotics, 2012.

[16] Z. Xu, T. Lü, H. Tian, Z. Xu, and L. Song, "Dynamic analysis of the biped ice-skater robot of passive wheel type," Journal of Shanghai Jiaotong University (Science), 2008.

[17] N. Ziv, Y. Lee, and G. Ciaravella, "Inline skating motion generator with passive wheels for small size humanoid robots," in IEEE/ASME International Conference on Advanced Intelligent Mechatronics (AIM), 2010.

[18] O. Matsumoto, S. Kajita, and K. Komoriya, "Flexible locomotion control of a self-contained biped leg-wheeled system," in IEEE/RSJ International Conference on Intelligent Robots and Systems (IROS), 2002.

[19] C. Iverach-Brereton, J. Baltes, J. Anderson, A. Winton, and D. Carrier, "Gait design for an ice skating humanoid robot," Robotics and Autonomous Systems, 2014.

[20] N. Takasugi, K. Kojima, S. Nozawa, Y. Kakiuchi, K. Okada, and M. Inaba, "Real-time skating motion control of humanoid robots for acceleration and balancing," in IEEE/RSJ International Conference on Intelligent Robots and Systems (IROS), 2016.

[21] R. W. de Boer and K. L. Nilsen, "The gliding and push-off technique of male and female olympic speed skaters," International Journal of Sport Biomechanics, 1989.

[22] C. D. Bellicoso, M. Bjelonic, L. Wellhausen, K. Holtmann, F. Günther, M. Tranzatto, P. Fankhauser, and M. Hutter, "Advances in real-world applications for legged robots," under review for Journal of Field Robotics, 2018

[23] M. Bloesch, H. Sommer, T. Laidlow, M. Burri, G. Nuetzi, P. Fankhauser, D. Bellicoso, C. Gehring, S. Leutenegger, M. Hutter, et al., "A primer on the differential calculus of $3 \mathrm{~d}$ orientations," arXiv preprint arXiv:1606.05285, 2016.

[24] C. Gehring, C. D. Bellicoso, S. Coros, M. Bloesch, P. Fankhauser, M. Hutter, and R. Siegwart, "Dynamic trotting on slopes for quadrupedal robots," in IEEE/RSJ International Conference on Intelligent Robots and Systems (IROS), 2015.

[25] A. Goldenberg, B. Benhabib, and R. Fenton, "A complete generalized solution to the inverse kinematics of robots," IEEE Journal on Robotics and Automation, 1985.

[26] M. Bloesch, C. Gehring, P. Fankhauser, M. Hutter, M. A. Hoepflinger, and R. Siegwart, "State estimation for legged robots on unstable and slippery terrain," in IEEE/RSJ International Conference on Intelligent Robots and Systems (IROS), 2013. 